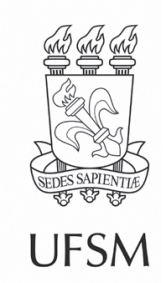

\title{
Artigos
}

\section{Caracterização anatômica da madeira de quatro espécies de Vochysiaceae conhecidas, na Amazônia brasileira, como "mandioqueira"}

\author{
Anatomical characterization of the wood of four species of Vochysiaceae, \\ known in the Brazilian Amazon as "mandioqueira"
}

\author{
Marta César Freire Silva' ${ }^{\bullet}$ \\ Fernanda Ilkiu Borges de Souza' ${ }^{\oplus}$ \\ Joaquim Ivanir Gomes ${ }^{1 \odot}$
}

'Embrapa Amazônia Oriental, Belém, PA, Brasil

\section{RESUMO}

Dentre as espécies madeireiras da Amazônia brasileira comercializadas devido a sua múltipla possibilidade de uso estão Qualea paraensis Ducke, Qualea acuminata Spruceex Warm., Qualea dinizii Ducke e Ruizterania albiflora (Warm.) Marc.-Berti, da família Vochysiaceae A. St.-Hil, todas conhecidas popularmente como "mandioqueira". O objetivo deste trabalho foi caracterizá-las sob o aspecto anatômico macroscópico e microscópico da madeira, dando ênfase às características qualitativas e quantitativas, visando subsidiar a identificação desse táxon. As espécies estudadas foram selecionadas no acervo da Xiloteca da Embrapa Amazônia Oriental e as descrições dos caracteres anatômicos seguiram as normas preconizadas em anatomia da madeira. As características quantitativas foram submetidas às análises estatísticas. A partir dos resultados foi possível distinguir as espécies por meio de características qualitativas (dos vasos, parênquimas e raios), e características quantitativas (dos vasos, raios e fibras), possibilitando aproximar o xilema secundário de Ruizterania albiflora ao de Qualea paraensis, com Qualea acuminata estando próxima às espécies, e Qualea dinizii se diferenciando das demais. Os resultados obtidos aqui evidenciam a anatomia da madeira como uma ferramenta importante a ser empregada na identificação e distinção confiáveis das espécies conhecidas como "mandioqueira", principalmente quando não se dispõe de materiais botânicos complementares.

Palavras-chave: Floresta amazônica; Anatomia do lenho; Potencial madeireiro 


\section{ABSTRACT}

Among the timber species from the Brazilian Amazon, commercialized due to their multiple possibilities of use, are Qualea paraensis Ducke, Qualea acuminata Spruce ex Warm., Qualea dinizii Ducke and Ruizterania albiflora (Warm.) Marc.-Berti, from the family Vochysiaceae A. St.-Hil., all popularly known as "mandioqueira". The objective of this work was to characterize them under the macroscopic and microscopic anatomical aspect of the wood, emphasizing the qualitative and quantitative characteristics, aiming to subsidize the identification of this taxon. The studied species were selected from the collection of Embrapa Amazônia Oriental Xylotheque and the descriptions of the anatomical characters followed the rules recommended in the wood anatomy. The quantitative characteristics were subjected to statistical analysis. From the results, it was possible to distinguish the species by qualitative characteristics (of the vessels, parenchyma and rays), and quantitative characteristics (of the vessels, rays and fibers), which allowed to approach the secondary xylem of Ruizterania albiflora to that of Qualea paraensis, with Qualea acuminate, being close of both species and Qualea dinizii differing from the others. The results obtained here highlight the wood anatomy like an important tool to be utilized on the reliable identification and distinction of the species known as "mandioqueira", mainly in the absence of other complementary botanical material.

Keywords: Amazon rainforest; Wood anatomy; Potential timber

\section{INTRODUÇÃO}

A Amazônia brasileira apresenta uma área de aproximadamente 4 milhões de $\mathrm{km}^{2}$, o que equivale a 49\% do território nacional. Estima-se que existam cerca de 60.000 espécies arbóreas. No entanto, a utilização comercial da madeira é limitada em aproximadamente 100 espécies, enquanto o número de madeiras exportadas para os mercados dos Estados Unidos (24\%), da Europa (44\%), da China (10\%) e de outros países (22\%) concentram-se em apenas 40 espécies (PEREIRA et al., 2010). Mesmo assim, o mercado madeireiro na Amazônia brasileira é um dos maiores do mundo, perdendo apenas para Malásia e Indonésia (ORGANIZACIÓN INTERNACIONAL DE LAS MADERAS TROPICALES, 2006). Especificamente no Estado do Pará, terceiro exportador de madeira do Brasil, atrás apenas do Paraná e Santa Catarina, foram os segmentos de madeira em tora, madeira serrada e produtos secundários de madeira (que englobam os setores da construção civil, moveleiro e de celulose e papel), que movimentaram no ano de 2018 quase 200 milhões de dólares (SISTEMA NACIONAL DE INFORMAÇÕES FLORESTAIS, 2019). A rentabilidade alcançada explica por que a atividade madeireira é vista como atrativa ao investimento privado de perfil tradicional (LEAL et al., 2018). 
Embora esses números sejam atraentes sob o ponto de vista econômico, não refletem o impacto ecológico da exploração sobre o recurso florestal, que se processa de forma predatória. Pivetta e Kanashiro (2002) afirmam que, por serem semelhantes a olhos não treinados, as espécies são confundidas e exploradas de forma desordenada e não sustentável, sendo a distinção das espécies de forma clara e didática necessária para minimizar prejuízos econômicos e colaborar para o controle da manutenção da biodiversidade. Portanto, a identificação botânica a partir de caracteres morfológico e anatômico é uma ferramenta valiosa, para aquisição de informações sobre uma espécie (MARCHIORI, 1995).

Durante o processo de identificação das espécies, a utilização da nomenclatura vernacular, nos inventários florestais e comercialização de madeira, pode conduzir a equívocos devido a algumas espécies apresentarem características organolépticas semelhantes. Tais erros, além de possibilitarem impactos ecológicos, podem levar a limitações de uso e trabalhabilidade do material, em virtude das diferenças nas propriedades físicas, químicas e mecânicas e, por essa razão, a nomenclatura científica, bem como a correta identificação anatômica oferecem maior segurança no ato da comercialização (MARTINS-DA-SILVA et al., 2014).

A Vochysiaceae A. St.-Hil. figura entre as famílias com maior riqueza de espécies na Amazônia, é constituída por cerca de 240 espécies distribuídas em oito gêneros Qualea Aubl., Ruizterania Marc.-Berti, Vochysia Aubl., Erisma Rudge, Salvertia St.-Hil., Callisthene Mart., Korupodendron Litt \& Cheek e Erismadelphus Mildbr., dos quais apenas seis possuem ocorrência comprovada no Brasil (ANGIOSPERM PHYLOGENY GROUP, 2009; JARDIM BOTÂNICO DO RIO DE JANEIRO, 2020). Embora algumas espécies de Qualea indiquem importância medicinal (SILVA; RABELO; ENOQUE, 2015), as avaliadas neste trabalho, conhecidas popularmente como mandioqueira: Qualea acuminata Spruceex Warm, Qualea dinizii Ducke, Qualea paraensis Ducke e Ruizterania albiflora (Warm.) Marc.-Berti (sinônimo homotípico = Qualea albiflora), não apresentam 
essa característica. Contudo, destacam-se por sua vasta comercialização e potencial madeireiro em diferentes usos econômicos, apesar de apresentarem caracteres estruturais semelhantes (LOUREIRO; SILVA, 1970).

As quatro espécies são nativas e não endêmicas no Brasil, distribuem-se pela Amazônia, em grande parte dos Estados da região norte, sendo que há ocorrência da Qualea paraensis no Estado do Mato Grosso (JARDIM BOTÂNICO DO RIO DE JANEIRO, 2020). Apesar de todas serem encontradas em florestas de terra firme, existem registros de Qualea acuminata em floresta de várzea e de Ruizterania albiflora em florestas ciliares (JARDIM BOTÂNICO DO RIO DE JANEIRO, 2020).

A madeira das espécies de Qualea, no geral, apresenta boa trabalhabilidade no que diz respeito à facilidade de tornear, acabamento e colagem. As madeiras de Ruizterania albiflora, Qualea acuminata e Qualea paraensis são indicadas para uso na construção civil, construção naval, setor moveleiro e de utilidade geral (LOUREIRO; SILVA, 1970; SOUZA; CAMARGOS, 2014). O potencial aproveitamento da madeira de Qualea dinizii para o setor moveleiro, materiais de acabamento, e de utilidade geral é apontado por Cardoso et al. (2012) e Souza e Camargos (2014). Reis et al. (2014) caracterizaram anatomicamente a madeira de dez espécies de Qualea, entretanto, nesse estudo, algumas características não foram apresentadas, fazendo com que houvesse a necessidade do desenvolvimento de novos trabalhos que aprofundassem a análise anatômica das espécies conhecidas como "mandioqueira”.

Assim, o objetivo deste trabalho é identificar caracteres de diferenciação anatômica das madeiras de Qualea acuminata, Qualea dinizii, Qualea paraensis e Ruizterania albiflora, a fim de propiciar maior confiabilidade de identificação e diferenciação de espécies madeireiras comercializáveis, contribuindo também para a diminuição da pressão sobre determinadas espécies florestais. 


\section{MATERIAL E MÉTODOS}

Para composição do estudo anatômico, com base nas características macroscópica e microscópica da madeira, foram selecionadas amostras de quatro espécies da família Vochysiaceae. De cada espécie foram selecionados três indivíduos a partir de amostras das duplicatas presentes no acervo da Xiloteca da Embrapa Amazônia Oriental, Belém, Pará, que possuíam material botânico equivalente registrado no Herbário IAN:

- Qualea acuminata Spruce ex Warm: (X-4723, IAN-136818); (X-4724, IAN158537); (X-7704, IAN-136653).

- Qualea dinizii Ducke: (X-4727, IAN-158471); (X-4728, IAN-159461); (X-4729, IAN-150549).

- Qualea paraensis Ducke: (X-4736, IAN-49015); (X-4737, IAN-59633); (X-4739, IAN-98517).

- Ruizterania albiflora (Warm.) Marc.-Berti: (X-4716, IAN-103117); (X-4717, IAN115401); (X-4719, IAN-148059).

As descrições macroscópicas foram realizadas em amostras de madeira, de acordo com Coradin e Muniz (1992), sendo consideradas as características organoléptica e anatômica, com visibilidade dos elementos estruturais a olho nu ou utilizando uma lente de aumento de até 10x.

As normas do IAWA Committee (1989) serviram como base para realização das descrições microscópicas da madeira. As lâminas histológicas de madeira foram confeccionadas a partir dos corpos de prova $(1,0 \times 1,5 \times 1,5 \mathrm{~cm})$, retirados do cerne das amostras selecionadas no acervo, foram submetidos ao pré-tratamento em autoclave à temperatura de $120^{\circ} \mathrm{C}$ e pressão de 1 ATM. Posteriormente, o processo de microtomia foi realizado para obtenção dos cortes histológicos nas seções transversal, longitudinal tangencial e longitudinal radial, com espessura de 18 a $25 \mu \mathrm{m}$. 
Os cortes histológicos foram submetidos a um processo de clarificação em uma solução de hipoclorito de sódio, e corados com safranina (BURGER; RICHTER, 1991). Em seguida foram desidratados em série alcoólica etílica crescente de 50\%, 70\%, 90\% e 100\% (JOHANSEN, 1940 apud KRAUS; ARDUIN, 1997), e imersos em acetato de n-butila, finalizando a montagem com Entelan, para obtenção de lâminas permanentes.

Para análise das dimensões das fibras e elementos vasculares, retiraram-se fragmentos do corpo de prova, que foram dissociados em solução de ácido acético glacial e peróxido de hidrogênio $(1: 1, v / v)$, pelo período de 24 horas em estufa à temperatura de $60^{\circ} \mathrm{C}$. Os tecidos dissociados foram lavados em água corrente e corados com safranina (FRANKLIN, 1945 apud KRAUS; ARDUIN, 1997). Após o processo de maceração, o material foi montado entre lâmina e lamínula com glicerina (PURVIS et al., 1964 apud KRAUS; ARDUIN, 1997).

As mensurações foram realizadas a partir de 30 medições para cada estrutura anatômica do lenho: fibras (comprimento, espessura total e lúmen), vasos (comprimento, diâmetro e frequência/ $\mathrm{mm}^{2}$ ), raios (altura, largura e frequência/ $\mathrm{mm}$ linear) e pontoações intervasculares, radiovasculares e parênquimo vasculares (diâmetro). Além disso, também foram contabilizados o número de células de parênquima axial contidas por série ocorrente e as porcentagens de tipos de vasos e raios. As características quantitativas passaram por teste estatístico de análise de variância (ANOVA), seguida do teste de normalidade de Shapiro-Wilk e teste Tukey LSD com nível de probabilidade de $a=0.05$. Os testes estatísticos foram conduzidos utilizando o software R (versão 3.6.0) (R CORE TEAM, 2019). A análise de agrupamento (Clustering Analysis) foi realizada por meio do software PAST (versão 4.02), utilizando as características anatômicas dos vasos (diâmetro, frequência/mm² e comprimento), dos raios (altura, largura, número de células e frequência) e das fibras (comprimento, diâmetro máximo, de lúmen e espessura e parede). As imagens macroscópicas e microscópicas foram capturadas utilizando estereoscópio e microscópio óptico com câmera acoplada, respectivamente. 


\section{RESULTADO}

\subsection{Características anatômicas qualitativas}

A análise macroscópica revelou que todas as espécies apresentavam porosidade com visibilidade a olho nu e difusa em arranjo tangencial (Qualea acuminata, Qualea dinizii e Qualea paraensis) e arranjo radial (Ruizterania albiflora). Não sendo observada distinção da camada de crescimento (Figura 1).

Figura 1 - Secções transversais vistas em aspecto macroscópico. A: Qualea acuminata. B: Qualea dinizii. C: Qualea paraensis. D: Ruizterania albiflora. Ar: Arranjo radial dos poros. At: Arranjo tangencial dos poros. Barras de escala: $1 \mathrm{~cm}$

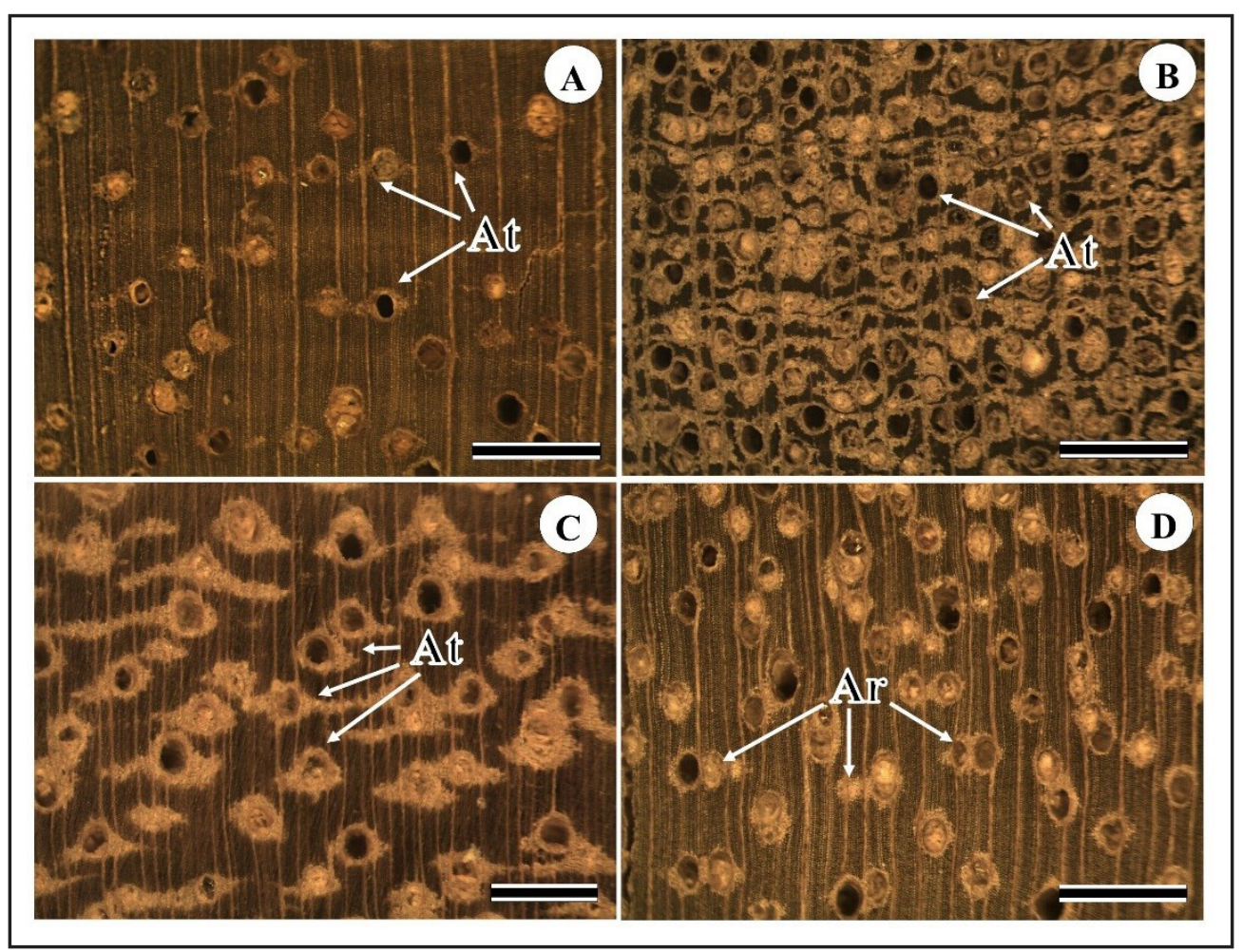

Fonte: Autores (2019)

De forma similar à camada de crescimento, o agrupamento de poros também foi o mesmo para todas as espécies, que apresentaram tanto vasos solitários como múltiplos de 2 a 4 células, contorno circular (Qualea acuminata, Qualea dinizii e Qualea 
paraensis) ou circular e ovalado (Ruizterania albiflora) (Figura 2), sendo ocasionalmente obstruídos por tilos. As espécies apresentaram vasos com placas de perfuração simples, pontoações intervasculares alternadas, pontoações radiovasculares e parênquimo vasculares, com aréolas distintas, similares às intervasculares em forma e tamanho, enquanto Qualea dinizii apresentou pontoações guarnecidas. No que diz respeito ao parênquima axial, Qualea acuminata, Qualea dinizii e Qualea paraensis apresentaram parênquima do tipo paratraqueal aliforme linear, com a possibilidade de formar confluências, ligando dois ou mais poros tangencialmente ou obliquamente (Figura 2). Ruizterania albiflora apresentou tanto parênquima paratraqueal do tipo aliforme losangular quanto vasicêntrico, sendo possível observar regiões de confluência ligando dois ou mais poros radialmente ou obliquamente (Figura 2).

Figura 2 - Secções transversais vistas em aspecto microscópico. A: Qualea acuminata. B: Qualea dinizii. C: Qualea paraensis. D: Ruizterania albiflora. Cf: Confluência de parênquimas. PAa: Parênquima axial aliforme. PAv: Parênquima axial vasicêntrico. Vm: Vaso múltiplo. Vs: Vaso solitário. Barras de escala: 600 m. Ampliação de A a D: 4x

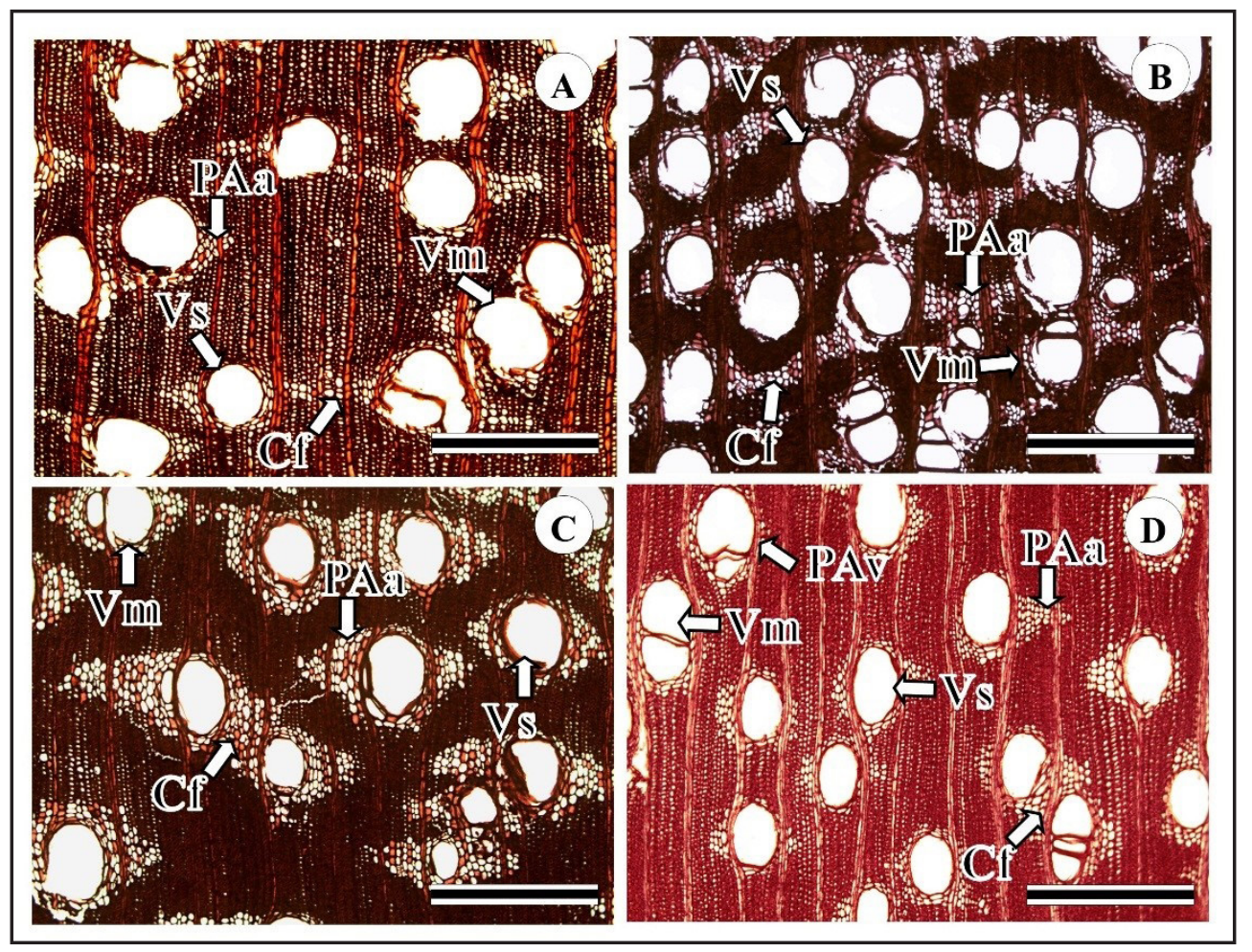

Fonte: Autores (2019) 
As quatro espécies apresentaram fibras libriformes com pontoações simples e não septadas. Quanto ao número de células nas séries de parênquima axial, Ruizterania albiflora apresentou de 3 a 4 células, já Qualea acuminata, Qualea dinizii e Qualea paraensis apresentaram séries com 3-4 e 5-8 células (Figura 3).

Figura 3 - Raios das quatro espécies estudadas, vistas em seção tangencial ( $A, B, C$ e D) e seção radial (E, F, G, H). A e E: Qualea acuminata. B e F: Qualea dinizii. C e G: Qualea paraensis. D e H: Ruizterania albiflora. Cp: Células procumbentes, Cq: Células quadradas, LV: Linha vascular. PA: Parênquima axial. R: Raios. Barras de escala de A a D, E e G: 600 um e F e H: 150 $\mu$ m. Ampliação de A a D, E e G: 4x e F e H: 10x

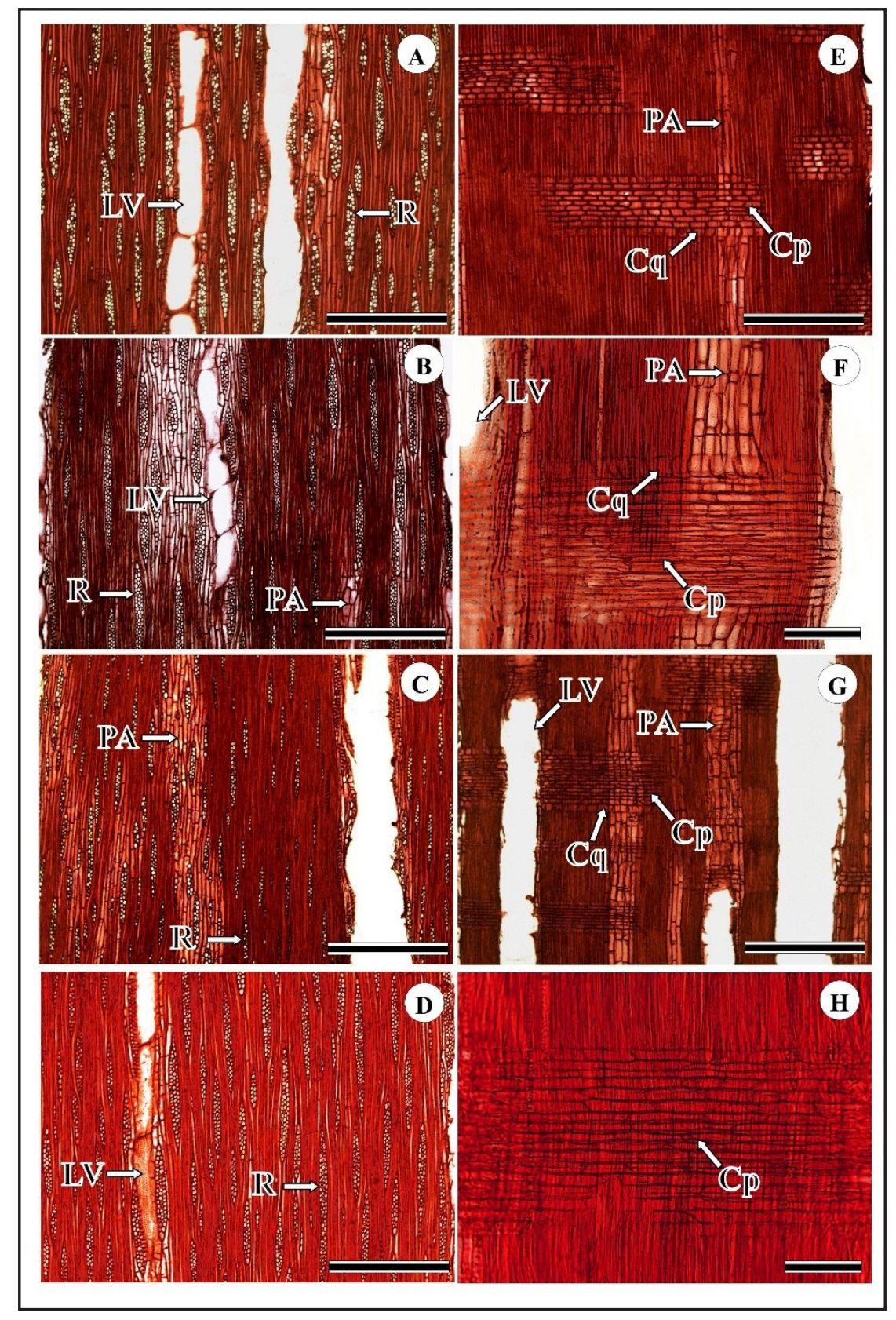

Fonte: Autores (2019) 
Os raios das quatro espécies são visíveis apenas com lente de aumento, não possuem estratificação aparente (Figuras 3A a 3D) e apresentam espelhamento contrastado. Além disso, os raios são agregados, sendo multisseriados com 1 a 3 células (Qualea acuminata, Qualea paraensis e Ruizterania albiflora) ou com 1 a 4 células de largura em Qualea dinizii (Figuras 3A a 3D). Na seção radial é possível observar dois tipos diferentes de raios: o primeiro do tipo heterogêneo formado por células procumbentes com uma camada marginal de células quadradas e está presente em Qualea acuminata, Qualea dinizii e Qualea paraensis (Figuras 3E a 3G), já o segundo, é homogêneo formado por células procumbentes, em Ruizterania albiflora (Figura 3H).

Não foram observados canais secretores ou variantes cambiais em nenhuma das espécies, entretanto, durante as avaliações no laboratório algumas inclusões minerais, como os cristais prismáticos (Qualea acuminata e Qualea dinizii) e corpúsculos silicosos (Qualea paraensis e Ruizterania albiflora) puderam ser localizados em células do parênquima axial e radial, respectivamente.

\subsection{Características anatômicas quantitativas}

A maior porcentagem de vasos solitários foi encontrada em Qualea paraensis (73\%) e a menor em Qualea dinizii e em Ruizterania albiflora (69\%), assumindo Qualea acuminata (70\%) posição intermediária. A porcentagem de vasos múltiplos seguiu tendência oposta, com maiores valores em Qualea dinizii e em Ruizterania albiflora (31\%). Não houve diferenças significativas entre as espécies tanto para o agrupamento de vasos solitários, quanto para os múltiplos (Figura 4A).

As espécies apresentaram elementos de vasos sésseis, bem como com apêndice em uma ou nas duas extremidades. Qualea dinizii apresentou a maior porcentagem de vasos sésseis (44\%), Qualea acuminata a maior porcentagem de vasos com apêndice em uma das extremidades (57\%), o que as diferenciou das demais estatisticamente (Figura 4B). Enquanto Ruizterania albiflora (47\%) e Qualea paraensis (44\%) apresentaram o maior valor de elementos de vasos com apêndices nas duas extremidades, demonstrando grande similaridade dessa característica (Figura 4B). 
Figura 4 - Características dos elementos de vaso de Ruizterania albiflora, Qualea acuminata, Qualea dinizii e Qualea paraensis. A: Agrupamento de vasos solitários e múltiplos. B: Tipos de apêndices apresentados pelas espécies

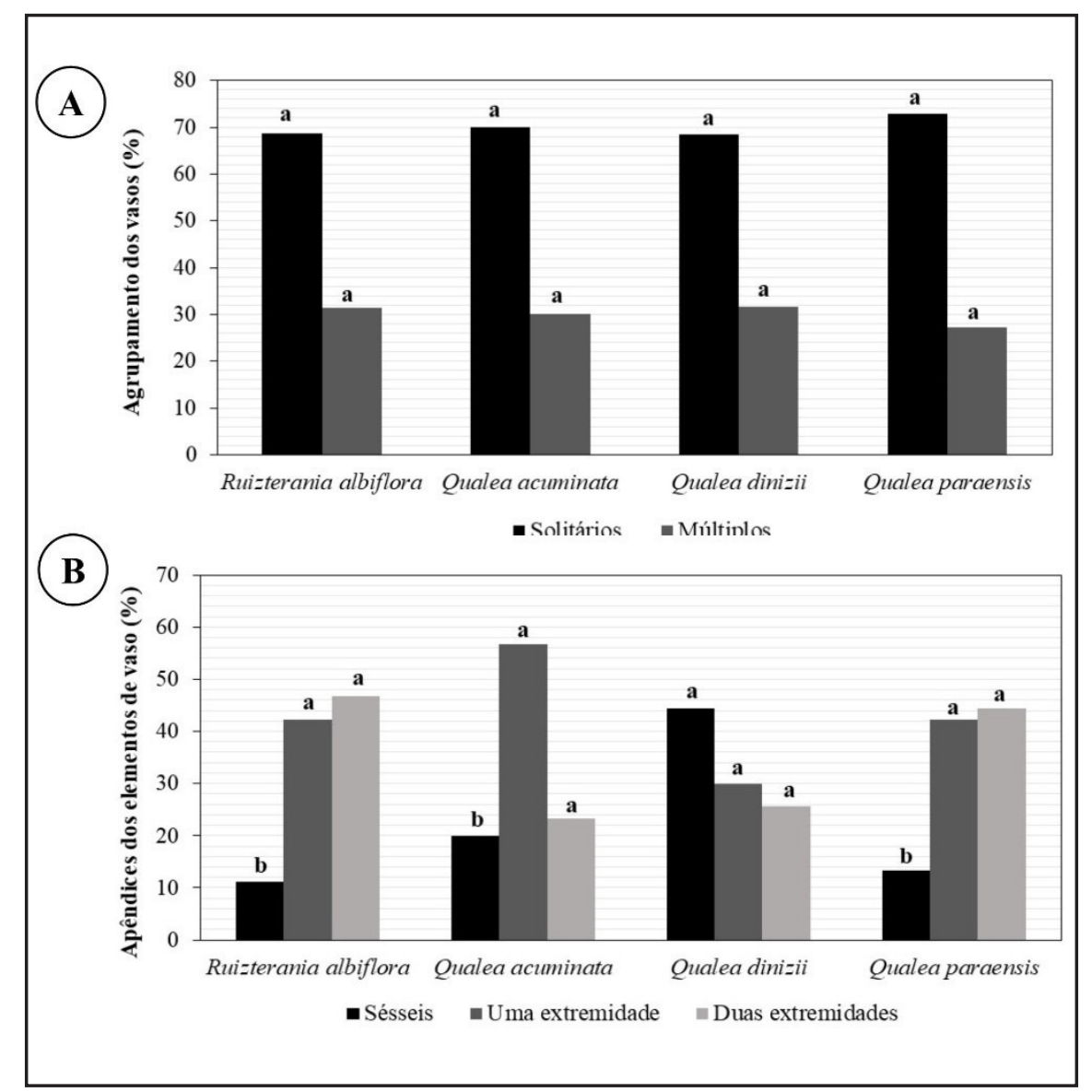

Fonte: Autores (2019)

Em que: (*) Letras iguais para as mesmas características avaliadas não diferem entre si pelo teste Tukey $(p<0,05)$.

Excetuando-se Qualea dinizii, que apresentou predominância de raios tetrasseriados, todas as outras espécies mostraram apenas raios unisseriados, bisseriados e trisseriados (Figura 5A). Não houve diferenças estatísticas entre as espécies para porcentagem de raios unisseriados. Tanto Qualea acuminata quanto Qualea paraensis se aproximaram estatisticamente pela predominância de raios bisseriados, diferenciando-se de Ruizterania albiflora e Qualea dinizii (Figura 5A). Já em Ruizterania albiflora, os raios trisseriados estiveram em maior quantidade, apresentando diferenças estatísticas quando comparadas a outras espécies (Figura 
5A). Como apenas Qualea dinizii apresentou raios tetrasseriados, isso a colocou em destaque nesta característica.

O intervalo médio para o diâmetro das pontoações intervasculares, radiovasculares e parênquimo vasculares esteve entre 6 e $7 \mu \mathrm{m}$, apresentando tamanho de pequeno a médio (Figura 5B). Ruizterania albiflora, Qualea dinizii e Qualea paraensis, além de apresentarem os maiores valores médios para pontoações radiovasculares, diferiram de Qualea acuminata nesta característica. Entretanto, não houve diferenças estatísticas consideráveis do diâmetro das pontoações intervasculares, radiovasculares e parênquimo vasculares entre as espécies (Figura 5B).

Figura 5 - Características dos raios e pontoações de Ruizterania albiflora, Qualea acuminata, Qualea dinizii e Qualea paraensis. A: Tipos de raios e respectivas porcentagens de ocorrência. B: Diâmetro das pontoações ocorrentes nas espécies

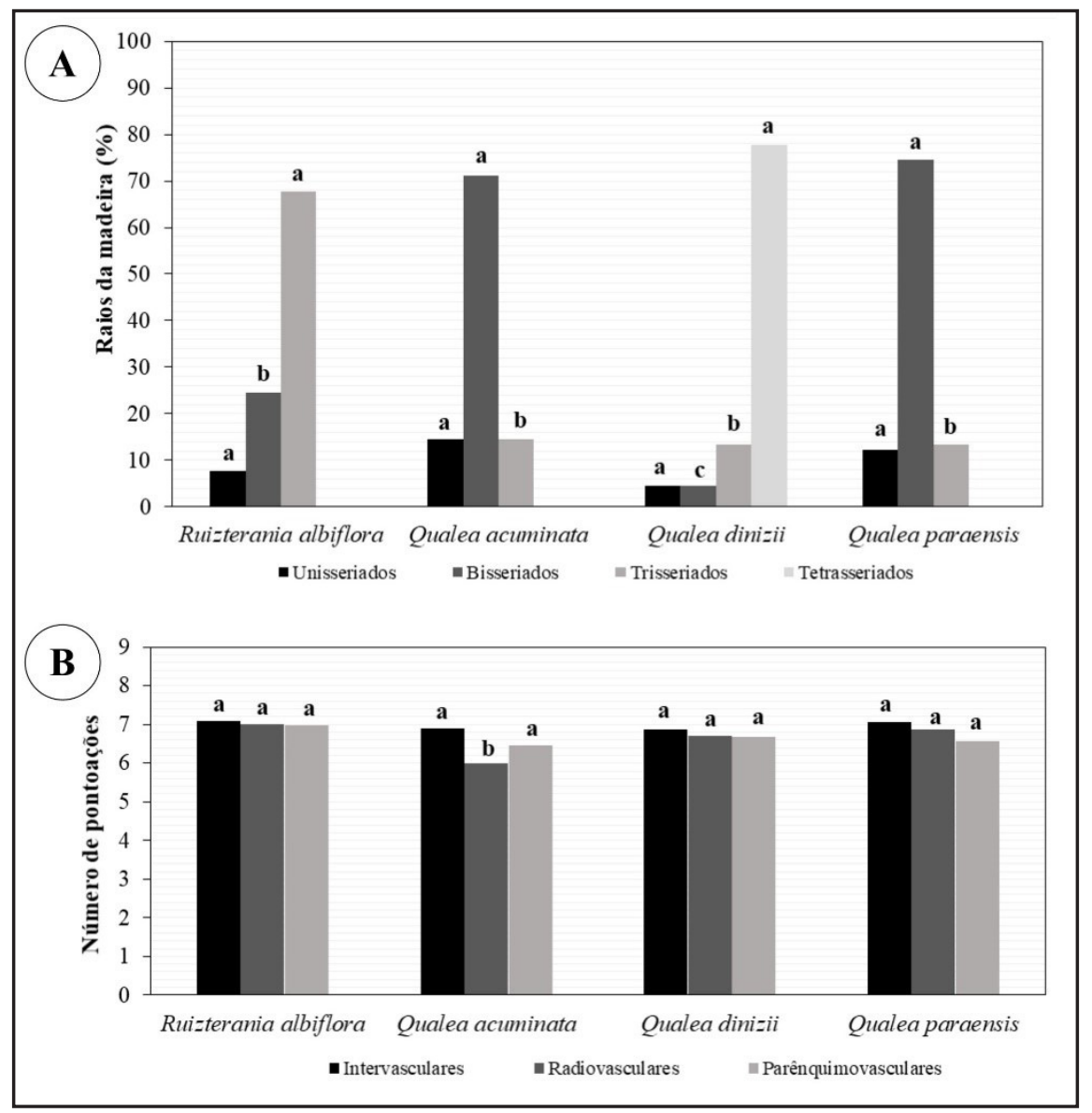

Fonte: Autores (2019)

Em que: $\left.{ }^{*}\right)$ Letras iguais para as mesmas características avaliadas não diferem entre si pelo teste Tukey $(p<0,05)$. 
Os maiores valores para diâmetro dos vasos foram visualizados em Qualea dinizii (204) e Qualea paraensis (202), seguidas de Qualea acuminata (199) e Ruizterania albiflora (197)(Tabela 1). Os valores médios do número de vasos por $\mathrm{mm}^{2}$ de Qualea dinizii (7), Ruizterania albiflora (7), Qualea paraensis (5) e Qualea acuminata (5) denotam um xilema secundário com poucos poros (Tabela 1). As espécies apresentaram vasos com comprimento curto a médio (Tabela 1) e Qualea acuminata apresentou maior valor médio de comprimento de vasos, estando a diferença para Qualea dinizii, de menor valor, na ordem de 19,65\%. Constatou-se que houve diferenças estatísticas nas características dos vasos avaliadas.

Tabela 1 - Biometria dos caracteres anatômicos das madeiras de Qualea acuminata, Qualea dinizii, Qualea paraensis e Ruizterania albiflora

\begin{tabular}{|c|c|c|c|c|}
\hline Características & $\begin{array}{c}\text { Qualea } \\
\text { acuminata }\end{array}$ & $\begin{array}{l}\text { Qualea } \\
\text { dinizii }\end{array}$ & $\begin{array}{c}\text { Qualea } \\
\text { paraensis }\end{array}$ & $\begin{array}{c}\text { Ruizterania } \\
\text { albiflora }\end{array}$ \\
\hline Altura dos raios (mm) & $\begin{array}{c}0,50 \text { b } \\
(0,46-0,57 ; 6,2)^{*}\end{array}$ & $\begin{array}{c}0,52 a \\
(0,47-0,60 ; 6,6)\end{array}$ & $\begin{array}{c}0,50 \mathrm{~b} \\
(0,46-0,57 ; 5,7)\end{array}$ & $\begin{array}{c}0,52 \mathrm{a} \\
(0,46-0,57 ; 5,5)\end{array}$ \\
\hline Altura dos raios em $\mathrm{n}^{\circ}$ de células & $\begin{array}{c}19 c \\
(13-32 ; 22,6)\end{array}$ & $\begin{array}{c}25 a \\
(20-30 ; 8,3)\end{array}$ & $\begin{array}{c}26 a \\
(20-33 ; 9,6)\end{array}$ & $\begin{array}{c}23 \mathrm{~b} \\
(2-27 ; 13,2)\end{array}$ \\
\hline Largura dos raios $(\mu \mathrm{m})$ & $\begin{array}{c}37,6 \text { b } \\
(30-50 ; 18,9)\end{array}$ & $\begin{array}{c}38,8 \text { bc } \\
(20-60 ; 18,9)\end{array}$ & $\begin{array}{c}27,1 \mathrm{c} \\
(20-40 ; 26,1)\end{array}$ & $\begin{array}{c}41,6 \mathrm{a} \\
(30-50 ; 16,1)\end{array}$ \\
\hline Largura dos raios em $n^{\circ}$ de células & $\begin{array}{c}2 c \\
(1-3 ; 18,9)\end{array}$ & $\begin{array}{c}4 a \\
(3-5 ; 15,7)\end{array}$ & $\begin{array}{c}2 c \\
(1-3 ; 18,3)\end{array}$ & $\begin{array}{c}3 b \\
(2-4 ; 16,7)\end{array}$ \\
\hline Frequência dos raios (mm linear) & $\begin{array}{c}4 c \\
(4-5 ; 10,7)\end{array}$ & $\begin{array}{c}4,5 \text { bc } \\
(3-6 ; 14,7)\end{array}$ & $\begin{array}{c}5 b \\
(4-6 ; 17,8)\end{array}$ & $\begin{array}{c}5,5 a \\
(4-7 ; 12,6)\end{array}$ \\
\hline Diâmetro dos vasos ( $\mu \mathrm{m})$ & $\begin{array}{c}199 a b \\
(170-250 ; 8,2)\end{array}$ & $\begin{array}{c}204 \mathrm{a} \\
(170-240 ; 6,2)\end{array}$ & $\begin{array}{c}202 \mathrm{a} \\
(170-230 ; 6,3)\end{array}$ & $\begin{array}{c}197 \text { b } \\
(170-230 ; 7,3)\end{array}$ \\
\hline Frequência dos vasos (vaso/mm²) & $\begin{array}{c}5 \mathrm{~b} \\
(2-8 ; 27)\end{array}$ & $\begin{array}{c}7 a \\
(3-13 ; 24,6)\end{array}$ & $\begin{array}{c}5 \mathrm{~b} \\
(2-8 ; 26,4)\end{array}$ & $\begin{array}{c}7 a \\
(3-12 ; 27,6)\end{array}$ \\
\hline Comprimento dos vasos ( $\mu \mathrm{m})$ & $\begin{array}{c}580 a \\
(200-900 ; 29,7)\end{array}$ & $\begin{array}{c}466 \mathrm{a} \\
(250-650 ; 19)\end{array}$ & $\begin{array}{c}539,4 a b \\
(300-830 ; 24,3)\end{array}$ & $\begin{array}{c}546,9 a \\
(300-750 ; 21,6)\end{array}$ \\
\hline Comprimento das fibras ( $\mu \mathrm{m})$ & $\begin{array}{c}1324 a \\
(1200-1500 ; 6,6)\end{array}$ & $\begin{array}{c}1314 a \\
(1200-1500 ; 6,8)\end{array}$ & $\begin{array}{c}1320 a \\
(1200-1500 ; 6,8)\end{array}$ & $\begin{array}{c}1332 \mathrm{a} \\
(1200-1500 ; 7,9)\end{array}$ \\
\hline Diâmetro máximo das fibras $(\mu \mathrm{m})$ & $\begin{array}{c}24,5 a \\
(15-37,5 ; 19,4)\end{array}$ & $\begin{array}{c}16,3 c \\
(7,5-25 ; 27,6)\end{array}$ & $\begin{array}{c}19,6 \text { b } \\
(12,5-25 ; 19,9)\end{array}$ & $\begin{array}{c}19,1 \mathrm{~b} \\
(12,5-30 ; 27,8)\end{array}$ \\
\hline Diâmetro lúmen das fibras ( $\mu \mathrm{m})$ & $\begin{array}{c}12,9 a \\
(5-25 ; 34,3)\end{array}$ & $\begin{array}{c}5,9 c \\
(2,5-12,5 ; 41,5)\end{array}$ & $\begin{array}{c}6,8 c \\
(2,5-10 ; 34,2)\end{array}$ & $\begin{array}{c}8,9 \mathrm{~b} \\
(2,5-17,5 ; 36,8)\end{array}$ \\
\hline Espessura parede das fibras ( $\mu \mathrm{m})$ & $\begin{array}{c}5,8 a b \\
(2,5-13,75 ; 38,3)\end{array}$ & $\begin{array}{c}5,2 \mathrm{ab} \\
(2,5-8,75 ; 31,6)\end{array}$ & $\begin{array}{c}6,4 a \\
(2,5-11,25 ; 31,2)\end{array}$ & $\begin{array}{c}5,1 \mathrm{~b} \\
(2,5-8,75 ; 33,7)\end{array}$ \\
\hline
\end{tabular}

Fonte: Autores (2019)

Em que: *Médias seguidas pelo intervalo entre mínimo e máximo e coeficiente de variação (\%) entre parênteses. Médias seguidas pela mesma letra a cada linha não diferem entre si pelo teste Tukey $(p<0,05)$. 
Apesar de apresentar o maior valor médio para comprimento de fibras, Ruizterania albiflora $(1332 \mu \mathrm{m})$ não diferiu estatisticamente das espécies de Qualea que apresentaram fibras de comprimento médio (Tabela 1). Qualea paraensis apresentou fibras com maior espessura de parede, entretanto, as fibras com maior diâmetro total foram encontradas em Qualea acuminata (Tabela 1).

Com relação às características dos raios, todas as espécies apresentam poucos raios, Qualea dinizii se diferenciou das demais espécies devido aos raios mais altos, quanto ao número de células contadas ao longo da altura dos raios, Qualea dinizii e Qualea paraensis apresentaram os maiores valores (Tabela 1). Ruizterania albiflora mostrou raios mais largos que as demais, porém Qualea dinizii foi a que apresentou maior número médio de células contados a partir da largura (Tabela 1). Os raios também foram mais frequentes em Ruizterania albiflora (Tabela 1).

\subsection{Agrupamento das espécies}

A análise de "clustering" permitiu o agrupamento das espécies por meio das dissimilaridades calculadas a partir da distância euclidiana (Figura 6). As características quantitativas avaliadas, ou seja, dos vasos (diâmetro, comprimento e frequência/ $\mathrm{mm}^{2}$ ), dos raios (altura, largura e frequência linear) e das fibras (comprimento, diâmetro máximo e do lúmen, espessura da parede), no geral aproximaram Qualea paraensis de Ruizterania albiflora. Qualea acuminata aparece relacionada ao grupo formado por Qualea paraensis e Ruizterania albiflora, enquanto Qualea dinizii apareceu distante das demais.

Figura 6 - Análise de agrupamento das espécies estudadas

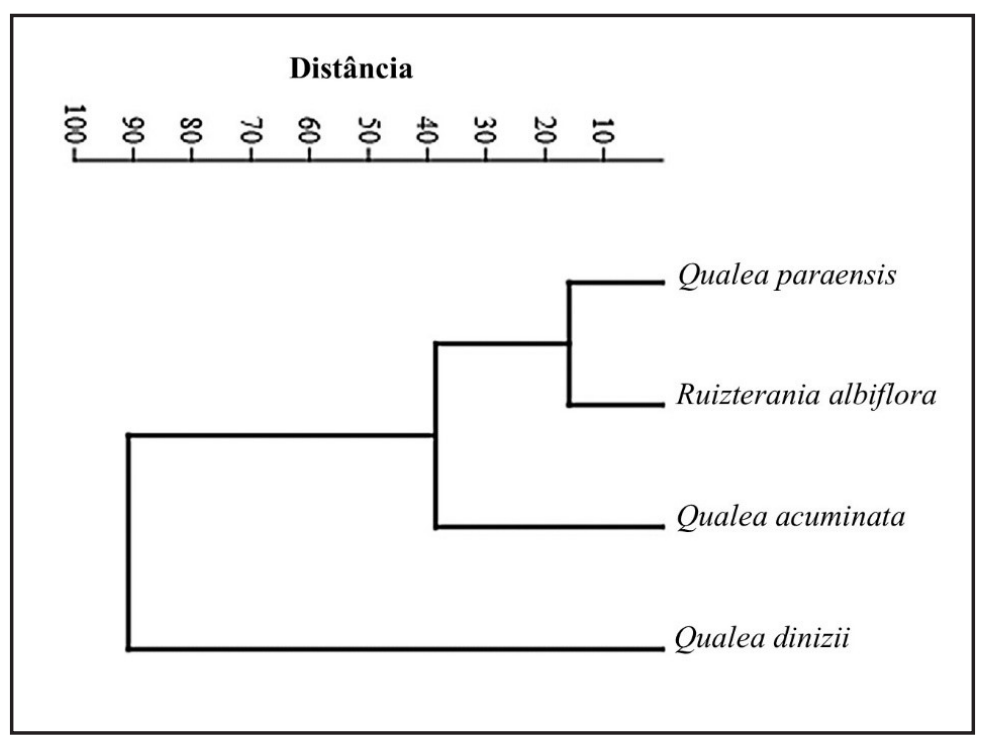

Fonte: Autores (2019)

Em que: *A escala corresponde à distância euclidiana. 


\section{DISCUSSÃO}

Ao se compararem os resultados obtidos é possível observar duas principais tendências. A primeira, a partir da avaliação dos caracteres qualitativos, aproximar as espécies de Qualea e as diferenciar de Ruizterania albiflora pelo arranjo e contorno dos vasos, bem como pelo parênquima axial, e pela composição celular dos raios. No entanto, se for levado em consideração a largura dos raios por número de células, não será possível diferenciar Qualea acuminata, Qualea paraensis e Ruizterania albiflora.

A segunda tendência diz respeito à análise dos caracteres quantitativos. A porcentagem de agrupamento de vasos, assim como o diâmetro de pontoações intervasculares, radiovasculares e parênquimo vasculares não se mostraram ideais para a diferenciação e separação anatômica entre as espécies. Enquanto a porcentagem de elementos vasculares que apresentaram apêndice séssil encontrada em Qualea dinizii a diferenciou das outras. Além disso, Cronquist (1988) e Baas et al. (2004) ressaltam que o conjunto de características anatômicas encontradas nos elementos de vaso tais como: elementos vasculares primitivos curtos que possuem apêndices sésseis, placa de perfuração simples, e pontoações alternas são indícios relacionados ao estágio evolutivo do vegetal e adaptação deste às condições ambientais,como encontrados em Qualea dinizii.

Mesmo que uma variação nas demais características quantitativas tenha sido observada, os valores médios aproximaram Qualea paraensis de Ruizterania albiflora. Quando comparados os valores médios obtidos por Qualea acuminata, observa-se maior proximidade de Qualea paraensis e de Ruizterania albiflora, do que de Qualea dinizii. Qualea dinizii, de fato, não esteve tão relacionada com outras espécies, por apresentar os maiores valores médios para grande parte das características quantitativas avaliadas (Tabela 1).

Dessa forma, se a avaliação anatômica for realizada com a observação das características quantitativas dos vasos e raios, elas podem auxiliar na distinção entre 
as espécies. Por exemplo, a altura dos raios em (mm) aproxima Qualea acuminata, Qualea paraensis e Ruizterania albiflora, entretanto, a largura dos raios em $(\mu \mathrm{m})$ pode separá-las, assim como o número de células contadas a partir da altura pode aproximar Qualea dinizii de Qualea paraensis e o número de células contadas pela largura, as separa. Se o objetivo for diferenciar Ruizterania albiflora das espécies de Qualea, observando os raios, a frequência por milímetro linear pode fornecer resultados satisfatórios, haja vista que os raios tendem a ser mais frequentes em Ruizterania albiflora. A predominância de um determinado tipo de raio também pode auxiliar na distinção entre as espécies, considerando-se que Ruizterania albiflora apresentou raios predominantemente trisseriados e Qualea dinizii tetrasseriados, e mesmo que Qualea acuminata e Qualea paraensis tenham apresentado raios predominantemente bisseriados, outras características deverão ser consideradas para a correta distinção entre as duas.

Do ponto de vista funcional, a quantidade de parênquima axial e radial pode ser determinante para a manutenção de processos que garantam a sobrevivência das plantas, uma vez que são compartimentos potencialmente disponíveis para o armazenamento de carboidratos não estruturais (PLAVCOVÁ; JANSEN, 2015), que podem ser acessados e transportados pelo sistema radial ou axial.

O parênquima axial que, segundo Pinheiro (1999), é característica de madeira com estrutura altamente especializada, juntamente com o parênquima radial visto na seção longitudinal tangencial, destacam-se como características macroscópicas de importante valor diagnóstico para identificação anatômica no campo, pois requerem a utilização de instrumento simples e acessível, obtendo resultados confiáveis, desde que tenham profissionais especializados na técnica de identificação macroscópica da madeira (BOTOSSO, 2011).

De acordo com IAWA Committee (1989), os diâmetros dos vasos das espécies avaliadas são classificados de médios a grandes (197-204 m), médios em comprimento (466 - $580 \mu \mathrm{m})$, sendo também poucos (5-7 vasos $/ \mathrm{mm}^{2}$ ) e em maioria, solitários, de 
69 a 73\% (Figura 4). Os vasos estão distribuídos de maneira uniforme, conferindo às madeiras das espécies maior resistência mecânica (BURGER; RICHTER, 1991).

Reis et al. (2014) analisando anatomicamente dez espécies de Qualea, entre elas Qualea dinizii, Qualea paraensis e Qualea albiflora (atualmente Ruizterania albiflora), observaram que apenas Qualea dinizii apresentou camadas de crescimento distintas por zonas fibrosas, além disso, todas as três espécies apresentaram poros visíveis e o arranjo tangencial foi também observado em Qualea dinizii, assim como o radial em Ruizterania albiflora, diferindo apenas o arranjo dos poros em Qualea paraensis, que tendeu ao diagonal.

Os valores médios encontrados por Reis et al. (2014) para comprimento e diâmetro dos vasos para Ruizterania albiflora estiveram no intervalo entre mínimo e máximo visualizado na Tabela 1. Já para Qualea paraensis e Qualea dinizii os valores médios encontrados pelos autores para diâmetro dos vasos estiveram acima do valor máximo encontrado aqui, sendo de 236,53 e 359, $25 \mu \mathrm{m}$, respectivamente. 0 comprimento dos vasos também salientado por Reis et al. (2014) para Qualea paraensis esteve dentro do intervalo estipulado na Tabela 1, porém Qualea dinizii, no trabalho dos autores, apresentou vasos mais curtos (aproximadamente $244 \mu \mathrm{m}$ ) do que o valor mínimo obtido aqui (Tabela 1).

Os vasos tendem a ter características anatômicas que respondem bem à disponibilidade hídrica (LONGUI et al., 2011; MELO JÚNIOR et al., 2017), e uma relação envolvendo comprimento, diâmetro e frequência dos vasos pode ser delimitada a partir desse parâmetro (BAAS et al., 2004). O dilema entre a eficiência e a segurança hidráulicas é conhecido em muitas espécies (MELO JúNIOR et al., 2017). Espécies com maior diâmetro e menor comprimento dos vasos investem mais em condução, porém estão sujeitas a condições de embolia e, por isso, a diminuição do diâmetro e aumento da frequência dos vasos é uma estratégia conhecida de segurança (BAAS et al., 2004; MELO JÚNIOR et al., 2017). Contudo, algumas espécies, dentre elas está Qualea dinizii, utilizam-se de outras estratégias, como a formação de pontoações guarnecidas 
para regulação da pressão osmótica, evitando a condição de embolia. Isso evidencia a plasticidade das espécies vegetais, demonstrando capacidade de adaptação a diferentes condições ambientais (BAAS et al., 2004; MELO JÚNIOR et al., 2017).

Pode ser atribuído às espécies aqui estudadas comportamento diferente devido a sua ocorrência em florestas de terra firme, várzea e matas ciliares da região amazônica (JARDIM BOTÂNICO DO RIO DE JANEIRO, 2020), onde a disponibilidade hídrica não é um fator limitante (BORDALO, 2017), e, portanto, não ocorrem modificações "bruscas" em suas estruturas de condução.

As quatro espécies apresentaram fibras libriformes, não septadas e médias (1314 a $1332 \mu \mathrm{m})$. Os valores médios para comprimento de fibras, espessura de parede e largura do lúmen encontrados por Reis et al. (2014), para Qualea dinizii, Qualea paraensis e Ruizterania albiflora estiveram dentro dos que foram encontrados para as amostras avaliadas neste trabalho (Tabela 1).

Para Castro e Silva et al. (1994), fibras libriformes conferem à madeira maior resistência mecânica, agregando valor às espécies no mercado madeireiro. De acordo com Melo Júnior et al. (2017), a espessura de parede das fibras tem influência sobre a densidade da madeira. Ribeiro e Zani Filho (1993) reforçam ainda que a densidade básica tem sido a característica mais utilizada na determinação da qualidade da madeira. A densidade, dessa forma, também pode ser aplicada para sustentar a viabilidade do uso da madeira na produção de móveis ou na construção civil (REZENDE et al., 2007). Ainda que, neste trabalho, a densidade da madeira das espécies não tenha sido determinada, fica claro que as características das fibras podem ser um indicativo do emprego preferencial da madeira das espécies de "mandioqueira" pelo setor da construção civil, naval e moveleiro (LOUREIRO; SILVA, 1970; CARDOSO et al., 2012; SOUZA; CAMARGOS, 2014).

Em comparação com as espécies de Qualea analisadas por Reis et al. (2014), encontramos valores similares para o intervalo de células que compõe as séries de parênquima axial, sendo 3-4 para Qualea dinizii e Ruizterania albiflora e 5-8 para Qualea 
paraensis. Quanto aos raios, as características observadas para Ruizterania albiflora não foram diferentes das encontradas por Reis et al.(2014), no entanto Qualea dinizii apresentou mais células nos raios (4 a 10) do que o intervalo visualizado no presente trabalho; o tipo de células também foi diferente para Qualea paraensis que apresentou raios homogêneos compostos por células procumbentes e Qualea dinizii com raios homogêneos de células eretas.

O caule, de fato, possui características sujeitas a alterações ambientais, que levam a explorar toda sua capacidade de adaptação expressa em sua plasticidade fenotípica (SILVA; MELO JúNIOR, 2017) e, portanto, as madeiras de Qualea acuminata, Qualea dinizii, Qualea paraensis e Ruizterania albiflora não são alheias a isso. Todavia, levando em consideração a distribuição dessas espécies limitada ao bioma amazônico (JARDIM BOTÂNICO DO RIO DE JANEIRO, 2020), infere-se que os dados apresentados neste trabalho não sejam distantes dos encontrados em análises anatômicas de outras amostras das espécies aqui avaliadas. Assim sendo, todas as características anatômicas observadas neste trabalho, mostram-se como confiáveis para a identificação e distinção entre Qualea acuminata, Qualea dinizii, Qualea paraensis e Ruizterania albiflora.

\section{CONCLUSÕES}

Uma detalhada caracterização anatômica de quatro espécies conhecidas como mandioqueira: Qualea acuminata, Qualea dinizii, Qualea paraensis e Ruizterania albiflora foi realizada. Não foram observadas diferenças significativas em relação às médias dos vasos (frequência/mm² e comprimento), pontoações intervascular, radiovascular e parênquimo vascular (diâmetro), raios (altura e frequência/mm linear) e das fibras (comprimento e espessura da parede). Contudo, os resultados permitiram diferenciar as espécies por características qualitativas, como o arranjo dos vasos, intervalo do número de células nas séries de parênquima axial e sua disposição, e pela composição celular dos raios; além das características quantitativas, como diâmetro do vaso, a predominância do tipo de apêndices dos elementos de vasos, a porcentagem de 
tipos de raios (bisseriado, trisseriado e tetrasseriado), altura (número de células) e largura (em $\mu \mathrm{m}$ e número de células) dos raios. Tais características permitiram a aproximação do xilema secundário de Ruizterania albiflora ao de Qualea paraensis, com Qualea acuminata estando próxima às espécies. Qualea dinizii pelas características quantitativas se distanciou das demais espécies.

As sutis diferenças encontradas entre as quatro espécies estudadas evidenciam a anatomia da madeira como uma importante ferramenta utilizada para fornecer uma correta identificação das espécies, principalmente na ausência de outros materiais botânicos.

\section{REFERÊNCIAS}

ANGIOSPERM PHYLOGENY GROUP III. An update of the Angiosperm phylogeny group classification for the orders and families of flowering plants: APGIII. Botanical Journal of the Linnean Society, London, v. 161, p. 105-121, 2009.

BAAS, P. et al. Evolution of xylem physiology. In: HEMSLEY, A. R.; POOLE, I. (ed.). The evolution of plant physiology: from whole plant to ecosystems. Amsterdam: Elsevier Academic, 2004. p. 273-295.

BORDALO, C. A. O paradoxo da água na região das águas: o caso da Amazônia brasileira. GEOUSP: Espaço e Tempo, São Paulo, v. 21, n. 1, p. 120-137, 2017.

BOTOSSO, P. C. Identificação macroscópica de madeiras: guia prático e noções básicas para o seu reconhecimento. Colombo: Embrapa Florestas, 2011. 65 p. (Embrapa Florestas. Documentos, 194).

BURGER, L. M.; RICHTER, H. G. Anatomia da madeira. São Paulo: Nobel, 1991. 154 p.

CARDOSO, C. C. et al. Caracterização físico-mecânica de madeiras amazônicas com aptidão tecnológica para comercialização. Revista Ciências Agrárias, Santarém, v. 55, n. 3, p. 176-183, 2012.

CASTRO E SILVA, A. et al. Noções básicas de anatomia de madeira. Manaus: Instituto de Tecnologia do Amazonas, 1994. 28 p.

CORADIN, V. T. R.; MUNIZ, G. I. B. Normas de procedimentos em estudos de na anatomia de madeira: Angiospermae e Gimnospermae. Brasilia: Ibama; LPF, 1992. 17 p.

CRONQUIST, A. The evolution and classification of flowering plants. New York: The New York Botanical Garden, 1988. 555 p. 
IAWA COMMITTEE. List of microscopic features for hardwood identification. IAWA, Leiden, v. 10, n. 3, p. 219-332, 1989.

JARDIM BOTÂNICO DO RIO DE JANEIRO. Flora do Brasil 2020 em construção: Vochysiaceae. Rio de Janeiro, 2020. Disponível em: http://floradobrasil.jbrj.gov.br/reflora/floradobrasil/ FB88376. Acesso em: 24 set. 2020.

KRAUS. J. E.; ARDUIN. M. Manual básico de métodos em morfologia vegetal. Rio de Janeiro: UFRRJ; EDUR, 1997. 198 p.

LEAL, S. R. et al. Atratividade do mercado madeireiro ao investimento privado no Estado do Pará. Revista Agroecossistemas, [s. I.], v. 9, n. 2, p. 299-307, abr. 2018.

LONGUI, E. L. et al. Caracterização do lenho e variação radial de Pittosporum undulatum Vent. (pau-incenso). Hoehnea, São Paulo, v. 38, n. 1, p. 37-50, 2011.

LOUREIRO, A. A.; SILVA, M. F. da. Contribuição para o estudo dendrológico e anatômico da madeira de três espécies de Qualea (Vochysiaceae) da Amazônia. Acta Amazônica, Manaus, v. 7, n. 3, p. 407-416, 1970.

MARCHIORI, J. N. C. Elementos da dendrologia. Santa Maria: Ed. UFSM, 1995. 163 p.

MARTINS-DA-SILVA, R. C. V. et al. Noções morfológicas e taxonômicas para identificação botânica. Brasília, DF: Embrapa, 2014. 103 p.

MELOJÚNIOR, J. C. F. de. Anatomia funcional da madeira e condutividade hidráulica de espécies lenhosas de restinga. In: MELO JÚNIOR, J. C. F. de; BOEGER, M. R. T. Patrimônio natural, cultura e biodiversidade da restinga do Parque Estadual Acaraí. Joinville: UNIVILLE, 2017. Cap. 8.

ORGANIZACIÓN INTERNACIONAL DE LAS MADERAS TROPICALES. Reseña anual y evaluación de la situación mundial de las maderas. Yokohama, 2006. 222 p.

PEREIRA, D. et al. Fatos Florestais da Amazônia 2010. Belém: Imazon, 2010. 126 p.

PINHEIRO, A. L. Considerações sobre a taxonomia, filogenia, ecologia, genética, melhoramento florestal e a fertilização mineral e seus reflexos na anatomia e qualidade da madeira. Viçosa, MG: UFV; DEF, 1999. 144 p.

PIVETTA, M.; KANASHIRO, M. As diferenças entre árvores. Pesquisa Fapesp, São Paulo, v. 82, p. 33, 01 dez. 2002.

PLAVCOVÁ, L.; JANSEN, S. The role of xylem parenchyma in the storage and utilization of nonstructural carbohydrates. In: HACKE, U. Functional and ecological xylem anatomy. Switzerland: Springer, 2015. p. 209-234.

R CORE TEAM. R: a language and environment for statistical computing. Vienna: R Foundation for Statistical Computing, 2019. Disponível em: https://www.R-project.org/. Acesso em: 08 jul. 2020. 
REIS, A. R. S et al. Caracterização anatômica da madeira de mandioqueira (Qualea Aubl.) comercializada no mercado madeireiro do Estado do Pará. Enciclopédia Biosfera, Goiânia, v. 10, n. 19, p. 448-462, 2014.

REZENDE, M. A. et al. Estudo das variações de densidade da madeira do Liquidambar styraciflua e do Pinus tecunumannii, como parâmetros de qualidade para a produção de móveis. Holos Environment, Rio Claro, v. 7, n. 1, p. 60, 2007.

RIBEIRO, F. A; ZANI FILHO,J. Variação da densidade básica da madeira em espécies/procedências de Eucalyptus spp. IPEF, Piracicaba, n. 46, p. 76-85, 1993. Disponível em: http://jmg.bmj.com/ lookup/doi/10.1136/jmedgenet-2012-101146. Acesso em: 11 ago. 2020.

SILVA, A. F.; RABELO, M. F. R.; ENOQUE, M. M. Diversidade de angiospermas e espécies medicinais de uma área de Cerrado. Revista Brasileira de Plantas Medicinais, Botucatu, v. 17, n. 4, p. 1016-1030, 2015.

SILVA, M. M.; MELO JÚNIOR, J. C. F. Plasticidade da folha e lenho de cinco espécies lenhosas em duas áreas de restinga no Sul do Brasil. Iheringia - Serie Botanica, Porto Alegre, v. 72, n. 2, p. 173-180, 2017.

SISTEMA NACIONAL DE INFORMAÇÕES FLORESTAIS. Produção, economia e mercado florestal, 2019. Brasília, 2019. Disponível em: http://snif.florestal.gov.br/pt-br/produtosflorestais-madeireiros-exportacao. Acesso em: 11 ago. 2020.

SOUZA, M. H. de; CAMARGOS, J. A. A. Madeiras Tropicais Brasileiras = Brazilian Tropical Woods. Brasília: SFB; LPF, 2014. v. 2. 150 p.

\section{Contribuição de Autoria}

\section{1 - Marta César Freire Silva}

Bacharel em Biologia, Assistente de Pesquisa

https://orcid.org/0000-0001-7473-0661・marta.silva@embrapa.br

Contribuição: Conceituação, Curadoria de dados, Análise Formal, Investigação, Metodologia, Administração do projeto, Escrita - primeira redação, Escrita - revisão e edição

\section{2 - Fernanda Ilkiu Borges de Souza}

Engenheira Agrônoma, Dra., Pesquisadora

https://orcid.org/0000-0002-2363-9224•fernanda.ilkiu@embrapa.br

Contribuição: Conceituação, Metodologia, Administração do projeto, Recursos, Supervisão, Escrita - revisão e edição 


\section{3 - Joaquim Ivanir Gomes}

Engenheiro Agrônomo, Me., Pesquisador Aposentado https://orcid.org/0000-0002-3934-8421•jig.1975@yahoo.com.br

Contribuição: Análise Formal, Metodologia, Escrita - revisão e edição

\section{Como citar este artigo}

Silva, M. C. F.; Souza, F. I. B.; Gomes, J. I. Caracterização anatômica da madeira de quatro espécies de Vochysiaceae conhecidas, na Amazônia brasileira, como "mandioqueira". Ciência Florestal, Santa Maria, v. 31, n. 3, p. 1193-1215, 2021. DOI 10.5902/1980509835831. Disponível em: https://doi.org/10.5902/1980509835831. Acesso em: xx mês-abreviado. 2021. 